\title{
Cryogenic Coherent X-ray Diffraction Imaging Techniques for Structural Analyses of Biological Cells and Cellular Organelles
}

\author{
$\underline{\text { Amane Kobayashi }}^{1,2}$, Yuki Takayama ${ }^{3}$, Tomotaka Oroguchi ${ }^{1,2}$, Koji Okajima $^{1,2}$, Mao Oide ${ }^{1,2}$, Masaki \\ Yamamoto $^{2}$ and Masayoshi Nakasako ${ }^{1,2, *}$ \\ 1. Department of Physics, Faculty of Science and Technology, Keio University, 3-14-1 Hiyoshi, \\ Kohoku-ku, Yokohama, Kanagawa 223-8522, Japan. \\ 2. RIKEN SPring-8 Center, 1-1-1 Kouto, Sayo, Hyogo 679-5148, Japan \\ 3. Graduate School of Material Science, University of Hyogo, 3-2-1, Kouto, Kamigori, Ako, Hyogo 678- \\ 1297, Japan. \\ * Corresponding author, email: nakasako@phys.keio.ac.jp
}

Biological cells have complicated and hierarchical structures with cellular organelles such as a nucleus, chloroplasts and mitochondria, each of which include a numerous number of biological molecules such as proteins, nucleic acids, and lipids. In addition, they are active only in a fully hydrated environment. One of ultimate goals of cell biology is to understand the spatial temporal development of living cells by visualizing whole intact structures inside cells. From this point of view, coherent X-ray diffraction imaging (CXDI) is one of desirable imaging methods because it has the potential to visualize a noncrystalline particle with micrometer to sub-micrometer size at a resolution of a few tens of nanometers without staining, sectioning, and chemical fixation. In CXDI experiments, an isolated specimen particle is irradiated by X-ray beams with high transverse coherence. From the Fraunhofer diffraction pattern recorded by an area detector, the electron density map of the particle projected along the direction of the incident X-ray is reconstructed by using the phase-retrieval algorithms. Recently, XFEL sources, which provide very intense X-ray pulses with almost complete coherence at a duration of tens fs, have been utilized in CXDI experiments. Only a single XFEL pulse enables us to collect a diffraction pattern from a sub-micrometer sized biological particle up to a resolution of better than $50 \mathrm{~nm}$ free from any radiation damage (diffraction before destruction) [1]. However, the XFEL pulse destroys the particle at the atomic level through the Coulombic explosion after the diffraction from the particle occurs.

We developed a diffractometer with a high-speed translation stage to provide fresh specimen particles into the irradiation area by raster-scanning specimens against the trains of XFEL pulses [2] (Fig. 1(a)). In our experiments at SACLA, silicon nitride membrane with $100 \mathrm{~nm}$ thickness, on which a large number of specimen particles are dispersed, is moved by stop-and-go motions in sync with XFEL pulses at the repetition rate of $30 \mathrm{~Hz}$. Because optical system is set in a vacuum chamber of the diffractometer to avoid X-ray absorption and scattering by air, we also developed methods for preparing frozen-hydrated biological specimens to keep functional structures of cells [3]. Furthermore, we proposed a procedure to estimate the spatial coherence of each focused XFEL pulse for the precise alignment of mirror optics [4] (Fig. 1(b)). For the efficient reconstruction of electron density maps from diffraction patterns, we improved phase-retrieval algorithms [5]. Nowadays, these developments allow us to collect $0.9 \sim 1.6$ million diffraction patterns in a beamtime and obtain a large number of electron density maps. In this talk, we will present our CXDI techniques (Fig. 1), and show recent results with respect to three-dimensional (3D) reconstruction of electron density maps of cyanobacterial cells by using the single particle analysis (unpublished, Fig. 2). 
References:

[1] H. N. Chapman et al, Nat. Phys. 2, (2006) 839-843.

[2] A. Kobayashi et al, J. Synchrotron Rad. 23 (2016) 975-989.

[3] A. Kobayashi et al, Rev. Sci. Instrum. 87 (2016) 053109 (15 pages).

[4] A. Kobayashi et al, Sci. Rep. 8 (2018) 831 (13 pages).

[5] A. Kobayashi et al, Optics Express 22 (2014) 27892-27909.

(a)

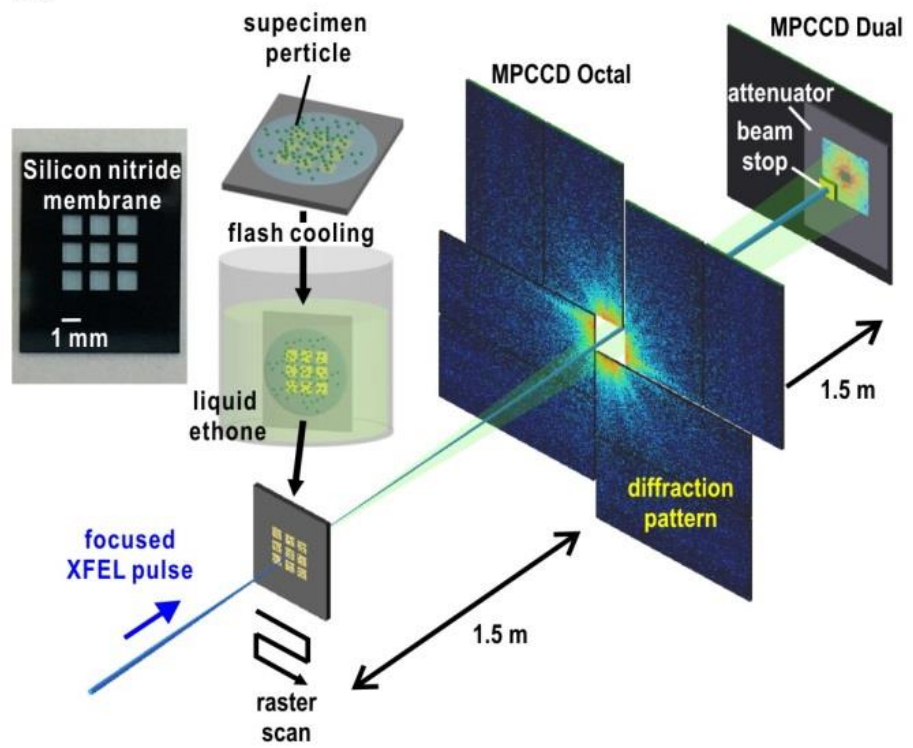

(b)

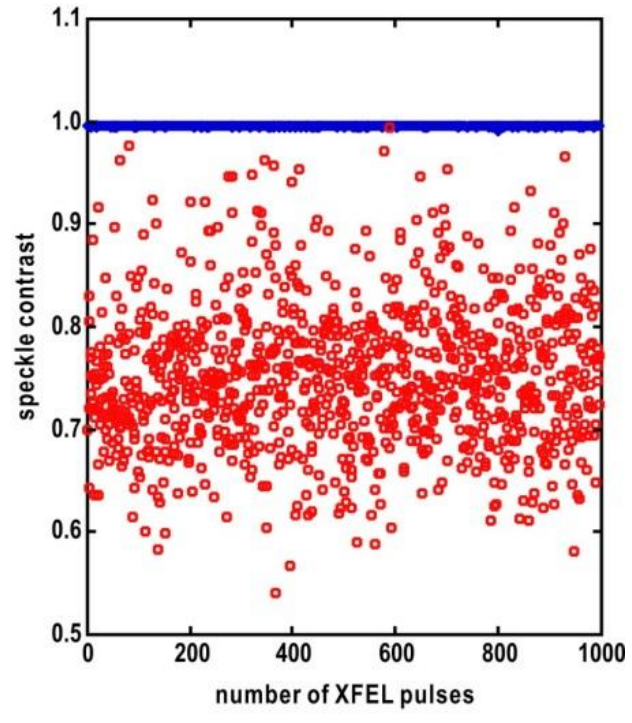

Figure 1 (a) Schematic illustration of our cryogenic XFEL-CXDI experiment. Biological specimen particles are flash-cooled to the frozen-hydrated state by using a liquid-ethane cooling device. (b) The variation of speckle contrast values, which is defined as square root of visibility of XFEL pulses. The blue dots represent that the focused XFEL pulses are almost full coherent light. However, when the focusing mirror optics is misaligned, the contrast values displayed a large fluctuation (red circles).
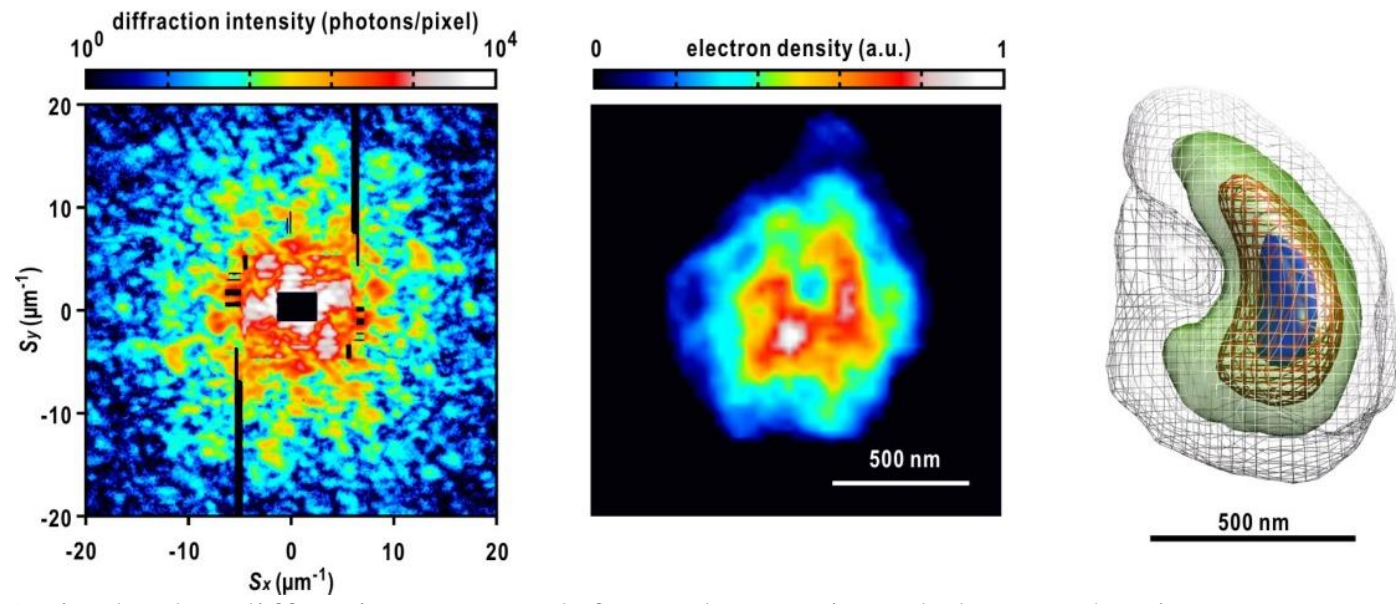

Figure 2 A single-shot diffraction pattern (left panel), a projected electron density map (center panel), and a 3D structure (right panel) of cyanobacterial cells, which was reconstructed from 1,733 projection maps. The surfaces of the 3D structure are contoured at 25\% (envelop), 40\% (green), 60\% (red), and 80\% (blue). C-shape structure viewed in the electron density map could be interpreted as a stack of thylakoid membranes with an abundance of protein. 